Brain and Cognition 40, 387-413 (1999)

Article ID brcg.1999.1087, available online at http://www.idealibrary.com on IDE $\mathbf{L}^{\circledR}$

\title{
Sentence Processing in Parkinson's Disease
}

\author{
Murray Grossman \\ Department of Neurology, University of Pennsylvania Medical Center
}

\begin{abstract}
Parkinson's disease (PD) is a neurodegenerative condition that is associated with the depletion of dopamine (DA)-containing neurons in specific brain regions. This article reviews one consequence of this defect-sentence comprehension difficulty in nondemented patients with PD. The first section describes the pattern of cognitive deficits seen in patients with PD, focusing specifically on their difficulties with language processing. Subsequent sections relate the profile of cognitive impairments in PD to studies investigating compromised DA metabolism in fronto-striatal brain regions. The findings suggest that the sentence comprehension deficit in PD is due in large part to limitations in the strategic distribution of cognitive resources such as selective attention that contribute to the processing of complex material. The physiological basis for this deficit appears to be associated with the disruption of a fronto-striatal cerebral network that is compromised following degradation of the DA projection system. ๑ 1999 Academic Press
\end{abstract}

\section{INTRODUCTION}

Parkinson's disease (PD) is a common neurodegenerative condition affecting the central nervous system that results in an akinetic-rigid movement disorder. The clinical diagnosis of this disease is based upon the presence of a quartet of motor signs: tremor, rigidity, gait disorder, and bradykinesia. The clinical features of PD are summarized in Table 1. In An Essay on the Shaking Palsy, James Parkinson (Parkinson, 1817) reported that "the senses and intellect are uninjured.' Nevertheless, more recent studies have demonstrated that many patients with PD exhibit a deterioration in mental function-

I am indebted to my colleagues Susan Carvell, Howard Geyer, Stephen Gollomp, Howard I. Hurtig, Julia Kalmanson, Jenifer Mickanin, Kris Onishi, Leticia Peltzer, Martin Reivich, Keith M. Robinson, Amanda Seidl, Matthew Stern, and Heather White for valuable help in conducting the studies described in this article. I also express my appreciation to Trish Giampapa for help in preparing this manuscript. Support was derived in part from the U.S. Public Health Service (NS35867 and AG15116) and the American Health Assistance Foundation.

Address correspondence and reprint requests to Murray Grossman, Department of Neurology, Hospital of the University of Pennsylvania, 3400 Spruce Street, Philadelphia, PA 191044283. Fax: 215-349-8464; E-mail: mgrossma@mail.med.upenn.edu. 
TABLE 1

Clinical Characteristics of Parkinson's Disease

Primary characteristics of Parkinson's disease

Tremor

Rhythmic, "pill-rolling" tremor involving the thumb and forefinger as well as other body segments that is slow (4 to $7 \mathrm{~Hz}$ ) and most prominent at rest but attenuated during intentional movements

Rigidity

Resistance to passive range of motion that is equally evident throughout the entire range of a joint's movement and regardless of the rate at which the joint is moved

Postural instability and gait abnormality

Stooped posture with drooped shoulders and flexed elbows and knees that is easily displaced backward due to impaired postural reflexes (retropulsion); small-stepped, shuf-

fling, "festinating"' gait without armswing that requires extra steps to turn and is associated with difficulty initiating and difficulty halting ambulation

Bradykinesia

Slowness and delay in executing motor acts without loss of motor power Secondary characteristics of Parkinson's disease

Reduced eyeblink rate

Hypominia (masked face)

Micrographia

Muscle cramps

Depression

Dysarthria

Dysphagia

Autonomic dysfunction

ing. Surveys conducted in our laboratory and elsewhere have documented that roughly $20 \%$ of patients with PD have a dementia (Mayeux et al., 1988, 1992; Ebmeier et al., 1991; Brown \& Marsden, 1984). Further, 20\% of PD patients appear to be normal intellectually. The remaining $60 \%$ of PD patients have an intellectual impairment that is less severe than a dementia, and includes difficulties with executive functioning and speed of mental information processing, a disorder of working memory, and a visual processing impairment (Owen et al., 1992; Jordan, Sagar, \& Cooper, 1992; Levin, 1993; Brown \& Marsden, 1988a,b,c). A representative cluster analysis of PD patients derived from their performance profile on a neuropsychological battery assessing attention, memory, visual processing, and executive functioning is illustrated in Fig. 1.

The following review focuses on language aspects of cognitive difficulty in nondemented patients with PD. Early investigations of language functioning reported equivocal results of language assessments in PD. Studies of single word use and sentence processing based on traditional aphasiologic measures at times described mild deficits (Geyer \& Grossman, 1994; Cummings, Darkins, Mendez, Hill, \& Benson, 1988), although difficulties in motor aspects of expression have been consistently noted. Most prominent among these have been descriptions of dysarthria (Illes, Metter, Hanson, \& 


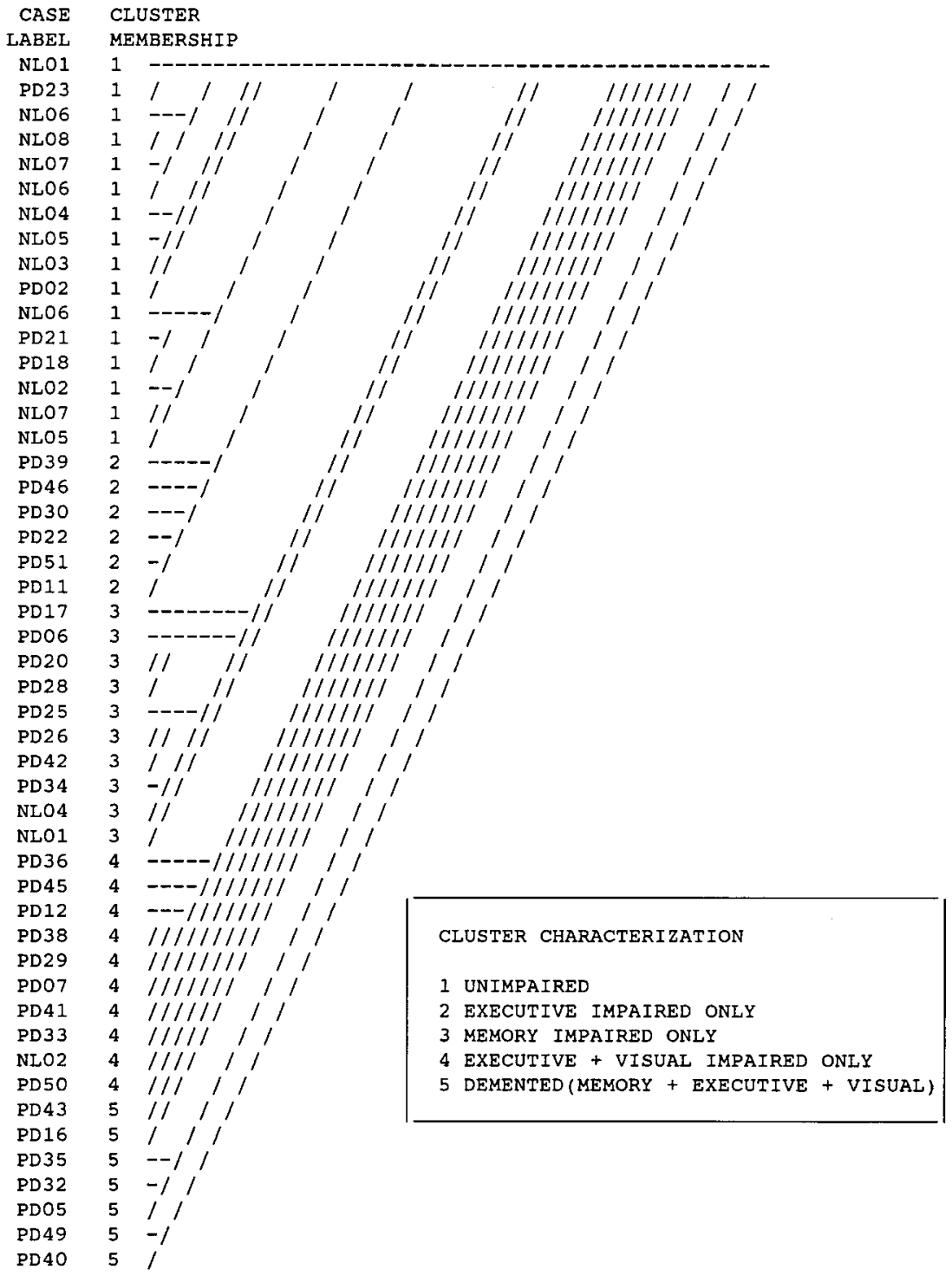

FIG. 1. Cluster analyses of Parkinson's disease patients based on their performance of neuropsychological measures of attention, memory, executive functioning, and visual processing. The clustering algorithm partitioned the entire population of subjects into subgroups based on their neuropsychological performance, as indicated by the horizontal and diagonal lines in the body of the figure leading to the individual subject IDs on the left margin of the figure. An ANOVA confirmed the differences in performance between the subgroups of PD patients $[F(5,44)=158.86$; $p \leq .0001]$. Case Label Prefix: NL, control subject; PD, Parkinson's disease. 
Iritani, 1988; Illes, 1990; Darkins, Fromkin, \& Benson, 1988). Confrontation naming difficulties and compromised category naming fluency have been reported by some investigators (Matison, Mayeux, Rosen, \& Fahn, 1982; Cooper, Sagar, Jordan, Harvey, \& Sullivan, 1991; Auriacombe et al., 1993). While some language difficulty has been evident, there has been universal agreement that PD patients do not have an aphasia that resembles one of the classic syndromic disorders of language seen after a stroke.

My collaborators and I initiated our investigation of sentence comprehension in PD in order to begin defining the role of so-called executive dysfunction and limited information processing speed in the language domain. Our studies thus were based on a model of sentence processing that includes both traditional linguistic components for computing the grammatical relationships between words in a sentence and processing components that facilitate appreciating a complex string of meaningful elements in a rapid and temporally constrained fashion. The latter set of processing components has been variously labeled "cognitive resources" or "working memory." In our approach to sentence processing, this component is thought to contribute to several aspects of sentence processing. For example, it may help regulate the distribution of attentional resources to specific aspects of sentences that are more demanding because of factors such as atypical mapping from a syntactic role to a thematic role. These atypical sentences may deviate from an expectation based on a frequency-based distribution of sentence frames. The mental manipulations required to process these demanding aspects of sentences, while ultimately governed by the syntactic relations specified in a grammar, also may require the allocation of additional computational resources. The relations specified in sentences containing subject-relative center-embedded clauses and object-relative center-embedded clauses are determined by the grammar, for example, but the on-line processing of the object-relative sentences takes longer than the subject-relative sentences at specific, relevant points in the sentence, and this extra time presumably reflects the additional demands associated with the atypical syntactic-thematic mapping found in object-relative clauses (Just \& Carpenter, 1992). Our conception of working memory in sentence processing thus is akin to Baddeley's (Baddeley, Logie, Bressi, Della Sala, \& Spinnler, 1986) central executive, but is distinct from a so-called phonological slave buffer that passively retains a finite amount of material-specific information in short-term memory for a limited period of time. The precise extent to which working memory depends on some absolute amount of cognitive resources or the efficient use of available cognitive resources is unclear at this point. Nor is it clear whether these cognitive resources are material-neutral in nature or are dedicated to specific aspects of language such as sentence processing. Nevertheless, we hypothesized that the appreciation of phrase structure and other complex, structural aspects of sentences would be selectively compromised in PD. We hoped to dissociate a disorder of syntactic processing per se from a cognitive 
impairment that happens to be associated with sentence level material. Specifically, we hypothesized that limitations in the distribution of attentional resources and deranged temporal integration of the recruited processing network contribute to sentence comprehension difficulty in PD.

We also focused our studies on the population of PD patients since we sought to understand the physiologic basis for sentence processing difficulty. Unlike patients with stroke, patients suffering from neurodegenerative conditions such as PD do not suffer from mixed lesions to neuronal populations and to white matter fibers that may not even be related to the critical neuronal population, but the lesion in PD is restricted to a specific neuronal defect mediated by a compromised dopamine (DA) projection system. Moreover, unlike many neurodegenerative conditions, the PD patients we studied do not suffer from a memory disorder or a dementia that can confound our interpretation of the sentence processing deficit. We thus sought to relate a specific sentence processing impairment to the relatively well understood neurotransmitter and neuroanatomical basis for PD, and particularly to the apparent involvement of a DA-mediated fronto-striatal network in the pathophysiology of PD. In sum, we hypothesized that the physiological basis for the sentence processing deficit in PD was the interruption of DA innervation of a fronto-striatal network. We hope to use these studies as a starting point for the development of rational intervention strategies for the treatment of sentence comprehension difficulty that can accompany many different disorders of the central nervous system.

\section{THE CHARACTERIZATION OF SENTENCE PROCESSING DIFFICULTY IN PARKINSON'S DISEASE}

Early studies in three independent laboratories demonstrated that patients with PD have a sentence comprehension deficit. Lieberman, Friedman, and Feldman (1990) administered the Rhode Island Test of Syntax to patients with idiopathic Parkinson's disease. This test requires patients to match a sentence containing a complex grammatical feature such as a relative clause with a picture in a forced-choice fashion. The sentences were designed so that there were no semantic or pragmatic constraints to support sentence interpretation, but instead required patients to decode the grammatical structure of the sentence. PD patients were significantly impaired on this task. Similarly, Natsopoulos et al. (1991) asked patients to perform a sentencepicture matching task. These investigators also found a significant deficit in PD patients.

In order to avoid confounds in visual processing that may be associated with impaired picture interpretation or difficulty mapping a syntactic role onto a picture, we asked PD patients to respond to a simple probe of an orally presented sentence (Grossman et al. 1991). The sentence stimuli varied along three dimensions: phrase structure, voice, and semantic constraint. 
Thus, the sentences were simple in structure, contained a right-branching or terminal subordinate phrase, or contained a center-embedded subordinate phrase. We found that sentences with center-embedded phrases are more difficult for PD patients than sentences with terminal subordinate phrases, and that both of these sentences are more difficult than simple sentences that do not have a subordinate phrase. A highly reliable order of difficulty was found in group-wide and individual patient analyses, with object-relative center-embedded sentences more error-prone than subject-relative centerembedded sentences. This confirmed previous results from other labs demonstrating a sentence comprehension deficit. To investigate the specificity of the sentence comprehension deficit in $\mathrm{PD}$, we manipulated semantic constraint as well. Thus, half of each type of sentence was constrained so that the subject and the object of the main verb could not be exchanged without violating commonly held assumptions about semantic relations in the world. The remaining items contained reversible subjects and objects. We found that PD patients are able to appreciate this type of semantic constraint. Finally, the voice of the sentence and the voice of the probe were manipulated so that half of the sentence-probe pairs had the same active or passive voice, while the remaining items had a different voice in the sentence and its probe. A grammatical impairment was expected to result in sentence-probe items cast in the passive voice to be more difficult than sentence-probe items cast in the active voice, but this was not found. Instead, we found greater difficulty with items that do not have a corresponding voice in the stimulus sentence and the probe compared to items with a corresponding voice in both the sentence and the probe. This was interpreted as consistent with an alternative hypothesis that limited cognitive resources contribute to PD patients' sentence comprehension difficulty.

A subsequent study (Grossman, Carvell, Stern, Gollomp, \& Hurtig, 1992) sought to replicate our initial results as well as to determine in greater detail the basis for the sentence comprehension deficit in PD. Thus, we examined another cohort of 20 PD patients on the set of sentences described above and performed additional assessments to test the role of short-term memory and selective attention in PD patients' sentence comprehension difficulty. In particular, the argument has been forwarded that limitations in short-term memory can compromise sentence interpretation since the buffer holding the sentence during linguistic processing can retain only a limited amount of information or can maintain the sentence information only for a limited period of time (Saffran \& Martin, 1990; Grossman, 1993; Caplan \& Waters, 1990). Short-term memory limitations such as these have been thought to contribute to deficits in sentence repetition and comprehension following stroke (Warrington \& Shallice, 1969; Caramazza, Basili, Koller, \& Berndt, 1981), although dissociations between auditory-verbal short-term memory and sentence comprehension also have been reported (McCarthy \& Warrington, 1987a; Luria, Sokolov, \& Klimkowski, 1967a). Some patients with 
PD have been found to have compromised short-term memory (Taylor, Saint-Cyr, \& Lang, 1990; Pillon, Deweer, Agid, \& Dubois, 1993; Orsini, Fragassi, Chiacchio, \& Falanga, 1987; Cooper, Sagar, \& Sullivan, 1993). We assessed the role of short-term memory in PD patients' sentence processing by correlating sentence comprehension with memory performance. However, we did not find any significant correlations. In addition, we evaluated sentence comprehension under a condition where elements of the main clause of a sentence were interrupted by a subordinate phrase, requiring the initial elements of the sentence to be held in short-term memory until the remainder of the main clause was encountered. However, probes of the main clause under this condition were no more difficult than probes of the main clause uninterrupted by a subordinate phrase.

This study also assessed the role of cognitive resources in sentence comprehension. Specifically, attentional resources must be selectively distributed to the crucial properties of a sentence and a mental manipulation of the sentence material must be performed in order to interpret the sentence appropriately. Consider the distinction between an active voice sentence and a passive voice sentence. At a surface level, the default interpretation of a simple transitive verb in an active voice English sentence licenses the assignment of the subject to the agent role and the object to the theme role. The presence of the verb morphology ". . . was -ed by ...' marks a passive voice, by comparison, generally indicating that the object of the prepositional phrase is the agent of the action named by the simple transitive verb. An appropriate distribution of attentional resources must be performed to detect this less typical verb form marking the unusual mapping from syntactic role to thematic role, and an appropriate realignment of syntactic roles and thematic roles must be executed. The appreciation of this atypical syntactic-thematic mapping thus may require a form of executive functioning (McCarthy \& Warrington, 1987b), and executive deficits such as compromised working memory and selective attention have been reported frequently in PD (Owen et al., 1992; Brown \& Marsden, 1988a,b). We assessed the role of these processing elements in PD patients' sentence comprehension difficulty by asking these patients to judge the correctness of sentences. Thus, sentences similar to the ones used in the comprehension experiment were designed to have errors, including an omitted obligatory grammatical morpheme, or a changed phonological shape of a grammatical morpheme. We also included errors involving a change in the position of a grammatical morpheme in a sentence that could be detected by a grammatical processing system but appeared to be less dependent on selective attention. PD patients experienced significantly more difficulty than control subjects at detecting a missing obligatory grammatical morpheme and at identifying an error in the phonological shape of a grammatical morpheme. However, PD patients were able to detect a word order error.

A stepwise linear regression analysis was performed to determine the rela- 
tive contribution of attentional, short-term memory, grammatical, and semantic factors to PD patients' overall sentence comprehension performance. The regression analysis identified a configuration of factors that explains $97.74 \%$ of the variance in PD patients' overall sentence comprehension performance. These variables (and their partial correlations) included the grammatical factors of subordinate phrase structure [0.658] and center-embedded phrase structure [0.694], the semantic variable of nonconstrained nouns [ -0.486$]$, and the selective attention variable of detecting the omission of an obligatory grammatical morpheme [0.819]. This regression formula was used to predict each PD patients' overall sentence comprehension performance, and the prediction was within $2 S D$ of every patient's actual performance.

We administered another sentence comprehension study to PD patients in order to evaluate the relative contribution of grammatical processing and attentional resource distribution more directly in PD patients. Geyer and Grossman (1994) thus focused on the appreciation of the linking rules by which thematic roles are mapped onto syntactic constituents. In languages such as English, thematic roles in a simple sentence are encoded most often by word order: The agent typically maps onto the subject and the theme usually maps onto the object. In most atypical mapping functions there are obvious surface markers, as in the morphology ". . . was -ed by . . ." in the passive voice. In comparison to the active voice, the passive voice focuses attention on the theme by stating this as the first noun in the surface manifestation of the sentence. This requires remapping subject-object and agent-theme relations in a sentence, but the cognitive resources needed for this are relatively modest since there is a surface marker to indicate the need for the remapping process. Indeed, our earlier studies indicated that the passive voice per se is not particularly difficult for PD patients to appreciate. In order to investigate the role of the executive system and resource distribution in sentence comprehension more closely, we contrasted the atypical syntactic-thematic linking rule of the passive voice with a sentence context where the canonical syntactic-thematic mapping function is not honored and is not signaled explicitly by surface markers. Specifically, this experiment exploited the distinction between simple transitive verbs and lexical causative verbs. In forming the intransitive entailment of a transitive sentence containing a simple transitive verb, the agent of the transitive sentence is the subject of the intransitive construction. Thus, "the girl applauded the clown" entails that "the girl applauded." By comparison, it is the object of a lexical causative verb in a transitive sentence that becomes the subject of the intransitive construction. "The woman drowned the swimmer' entails that "the swimmer drowned" and not that "*the woman drowned." The object of a lexical causative verb thus is the agent of the action in the active voice when the verb is used intransitively.

Grammatical and executive resources were directly contrasted in this ex- 
periment by embedding simple transitive and lexical causative verbs in a periphrastic sentence frame. The periphrastic frame neutralizes the differences between simple transitive and lexical causative verbs by making transparent the agent and theme relationships within a sentence. Thus, in a periphrastic sentence containing a simple transitive type of verb like "the comedian made the audience laugh" and in periphrastic sentences containing a lexical causative type of verb like "the woman made the swimmer drown," it is clear from the syntax which noun phrase is the agent and which the theme, that is, regardless of the type of verb. While syntactic-thematic mapping is transparent with the periphrastic sentence frame, these items are syntactically more demanding than active and passive sentences because of the subordinate phrase and the additional matrix verb. A grammatical processing impairment thus should yield impaired performance on periphrastic sentences regardless of the type of verb, while patients with a sentence comprehension deficit due to limited attentional resources should benefit from a periphrastic frame when interpreting a sentence that contains a lexical causative verb.

We found that PD patients have significantly greater difficulty interpreting sentences with lexical causative verbs than those with simple transitive verbs. We attributed this to the less canonical set of syntactic-thematic linking rules associated with lexical causative verbs compared to simple transitive verbs. We confirmed the hypothesis that limitations in distributing cognitive resources contribute to the sentence comprehension difficulty of PD patients by evaluating their appreciation of periphrastic sentences. Specifically, PD patients were more successful at interpreting periphrastic sentences with lexical causative verbs than active voice sentences with lexical causative verbs, that is, despite the increased grammatical complexity associated with the periphrastic sentences. Sentence processing difficulty in PD thus appeared to be related in part to limitations in the distribution of the cognitive resources needed to interpret sentences.

Lieberman et al. (1992) have performed detailed analyses of speech production in PD, showing that the phonetic characteristics of PD patients' speech output is very similar to that of Broca's aphasics. On the basis of this observation and its cooccurrence in patients with sentence comprehension difficulty, these investigators argued that grammatical aspects of sentence processing are compromised in PD. However, analyses of spontaneous expression in PD have not documented frankly agrammatic speech (Illes, 1990; Illes, Metter, Hanson, \& Iritani, 1988). Spontaneous speech expression is entirely under the control of the subjects' internal processing mechanism, and we cannot rule out the possibility that PD patients are producing correct but simplified grammatical forms because they are sensitive to their own sentence processing limitations. An alternative hypothesis is that limitations in working memory resources also contribute to sentence expression difficulty in PD. From this perspective, deficits in sentence expression may be- 
come evident as a function of the cognitive resource demands associated with the formulation of demanding grammatical relations, such as those involving atypical syntactic-thematic mapping.

To circumvent the problems associated with the patient's potential to simplify internally generated spontaneous speech, we asked PD patients to complete sentences that were constrained by both the context of the sentence and a picture they were asked to describe. In one study (Grossman, Carvell, \& Peltzer, 1994), PD patients were shown pictures of two receptacles containing different amounts and different types of substances. In particular, one container held a mass substance and the second container held a count substance. We tested grammatical subcategorization in a context with low resource demands, that is, by asking PD patients to complete a sentence with a single mass or count quantifier. The experimenter thus might say "This container has many (pointing appropriately to one of the pictured stimuli), but the other container (pointing again) has . . .' If the container held a small number of pencils, the correct response might be "few," if the container held a large amount of water, the correct response might be "much.", This evaluation of sentence completion failed to reveal a significant difference between PD patients and control subjects.

If cognitive resource limitations constrain sentence processing, however, we might expect to see greater difficulty on a task where PD patients are asked to produce a more demanding segment of a sentence. To test this possibility, we administered another sentence completion task to PD patients. Each item consisted of a picture and a related lead-in sentence. A second sentence about the picture was initiated by the experimenter, and patients were asked to complete this second sentence. The partial second sentence was structured so that it left off at a point where the patient was required to produce a particular grammatical form to describe the picture stimulus accurately (e.g., while presenting a picture of a bear chasing some children around a campground, the experimenter would say: "During their camping trip, the children had a frightening experience. The children ...' The patient was expected to respond: “. . . were chased by a bear'). Nine items attempted to elicit bound grammatical morphemes in simple phrase structure contexts, such as the plural '-s', the regular past tense '-ed,' and the irregular past (e.g., 'fell'). Seven items elicited members of a particular form class in simple phrase structure contexts, including a noun (e.g., 'painter'), an adverb (e.g., 'slowly'), and an adjective (e.g., 'taller'). Five items were designed to elicit responses requiring grammatical phrase attributes such as the passive voice, a question, and a subordinate phrase. During one presentation of the test items, the lead-in sentence and the partial second sentence were presented orally, and patients were asked to respond orally. The responses were tape-recorded, and we scored the most accurate response if a patient made several attempts at an item. During a second presentation of the test items, patients were asked to respond in writing to written material. The modalities 
of response were elicited in a randomly ordered fashion. Two independent raters agreed in $93.7 \%$ of their evaluations of the 20 PD patients' responses, and discrepancies were resolved by consensus discussion.

A MANOVA with a group (2) $\times$ modality $(2) \times$ type of item (3) design revealed only a significant group $\times$ type of item interaction $[F(2,84)=3.12$; $p<.03]$. Thus, PD patients were not more impaired than control subjects in their overall sentence completion. However, as shown in Fig. 2, PD patients differed significantly from control subjects in their attempts to complete sentences requiring more demanding grammatical material such as a subordinate phrase or the passive voice $[t(28)=2.54 ; p<.01]$. Within the PD group, patients encountered significantly more difficulty completing these items than items with bound grammatical morphemes $[t(19)=2.04 ; p<.05]$ or items from a particular major form class $[t(19)=2.15 ; p<.05]$. Similar patterns were seen for both oral and written response modalities.

We analyzed the nature of the errors produced by PD patients when they had difficulty completing sentences with more demanding grammatical material such as those requiring the passive voice. A MANOVA with a group (2) $\times$ modality (2) $\times$ type of error (4) design revealed a significant main effect for group $[F(1,28)=5.41 ; p<.03]$ and a significant group $\times$ type of error interaction $[F(3,84)=5.27 ; p<.005]$. As can be seen in Fig. 3, PD patients produced significantly more grammatical errors on these items than control subjects. Figure 3 also shows that, within the PD group, patients made significantly more grammatical errors than semantic errors $[t(19)=$ $4.63 ; p<.001]$, phonemic/graphemic errors $[t(19)=4.76 ; p<.001]$, or "other" errors such as mechanical and punctuation mistakes $[t(19)=4.33$; $p<.001]$. Table 2 provides examples of the errors produced by PD patients. Unlike agrammatic aphasics, their impairment did not result in telegraphic speech and writing. PD patients instead apparently have particular difficulty continuing the sentence in a fully coherent fashion. This appears to correspond to the comprehension errors seen in PD and is consistent with the claim that capacity limitations can have an effect on both comprehension and production.

The pattern of results on several of the studies described above have provided indirect support for the hypothesis that a limitation distributing cognitive resources contributes to sentence processing difficulty seen in PD. We assessed resource limitations in PD patients' sentence processing more directly by modifying a technique used by Baddeley Logie, Bressi, Della Sala, and Spinnler (1986) to investigate the central executive component of working memory. The theory underlying these "dual'" tasks is that there should be a decrement in performance when two tasks are performed synchronously because of the need to distribute cognitive resources across the two tasks. In subjects with limited resources, however, the decrement in performance under dual-task conditions should be greater, even if performance on each of the tasks is adequate when performed alone. Our present study independently 

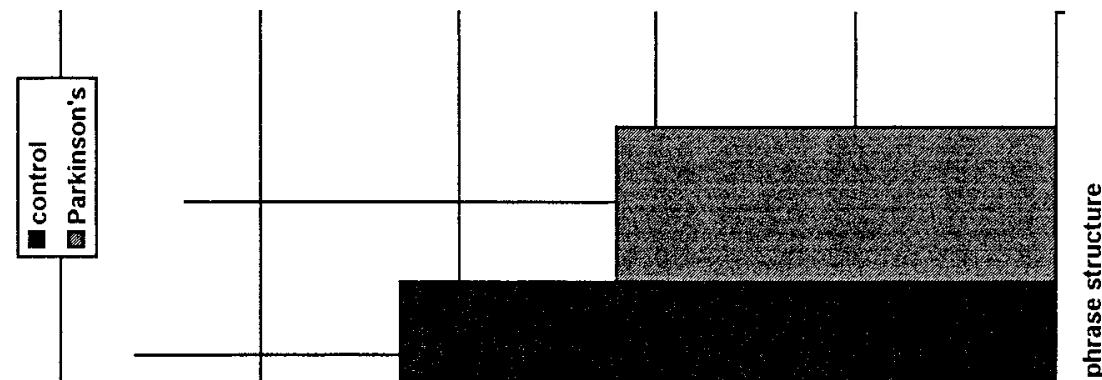

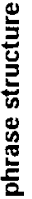
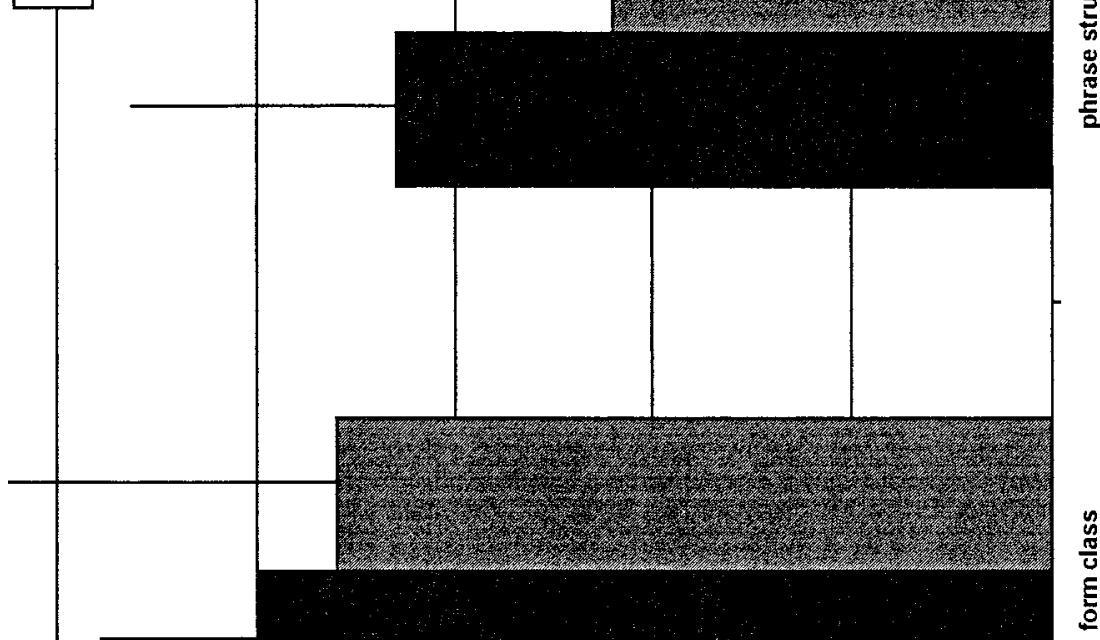

告
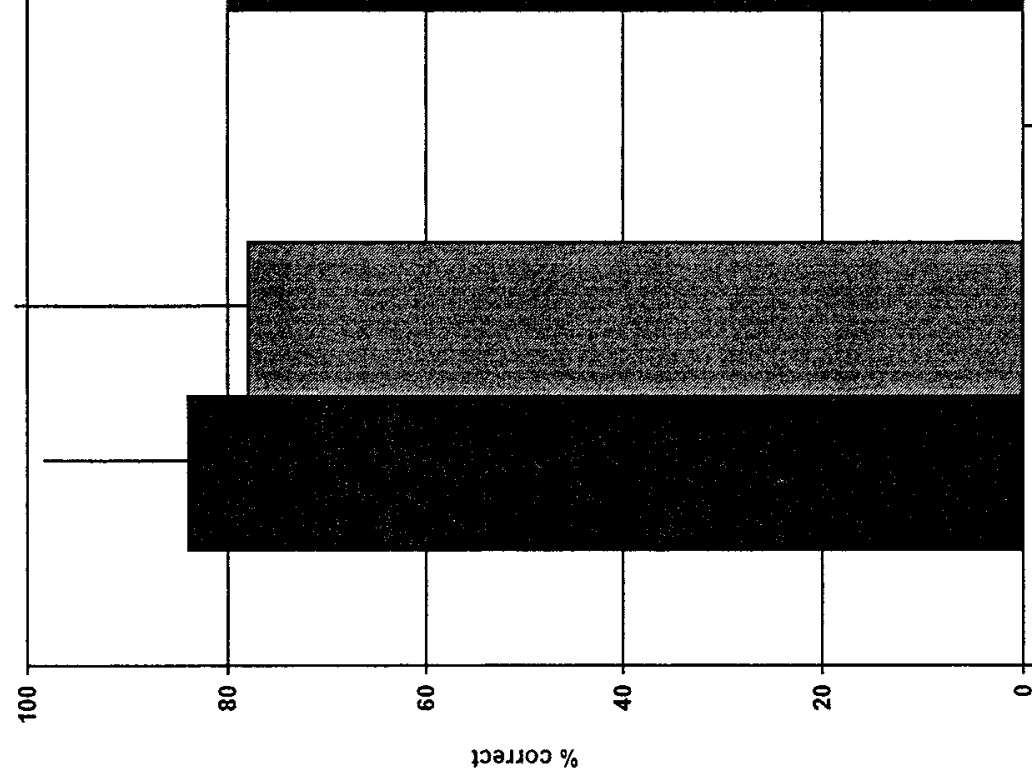

\%

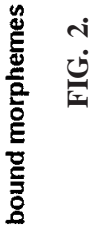




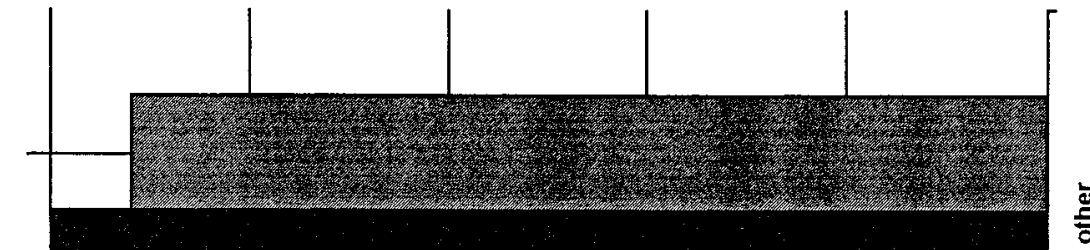

$\frac{}{0}$
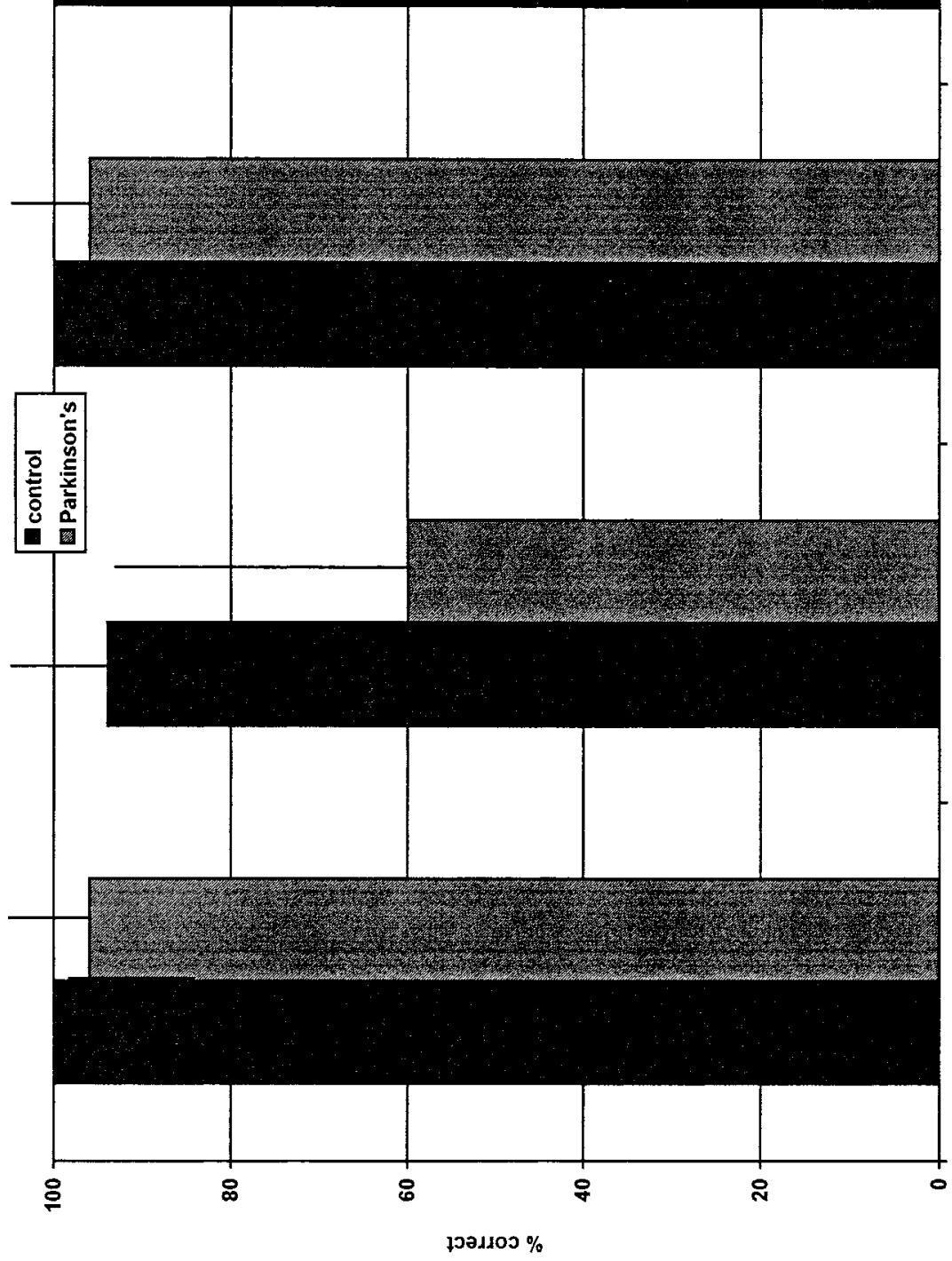

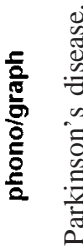


TABLE 2

Examples of Sentence Completion Errors in Parkinson's Disease

Oral sentences

Bob was injured during the baseball game. When he wasn't looking, he . . . "the ball hit him,

When the woman came home and found the broken lamp, she guessed that the lamp ... "fell from the cat",

During their camping trip, the children had a frightening experience. The children . . .

"ran in danger of their live from a bear"

Mr. Smith arrived at the bus stop 5 minutes late. He wondered if he had missed his bus, so he asked a woman, 'Pardon me ...' "'are the bus late"'

Grandma bakes unusual pies. Most pies are round, but the pies . . . "is square" Written sentences

Bob was injured during the baseball game. When he wasn't looking, he . . . "the ball hit him"

When the woman came home and found the broken lamp, she guessed that the lamp ... "cat broke the lamp",

During their camping trip, the children had a frightening experience. The children . . .

"chased by a bear",

My sister likes to exercise all the time. Instead of diriving to work, she . . " "ride her bicycle',

manipulated the resource demands of both the primary, sentence comprehension task and the secondary task. In this fashion, we could establish whether a specific processing system has limited cognitive resources.

The primary task involved answering a simple probe about orally presented sentences that were simple, contained a subject-relative centerembedded clause, or contained an object-relative center-embedded clause. These sentences, controlled for word length through the addition of filler adjectives, included a simple transitive action verb with a male name and a female name arrayed equally often in the subject position or the object position. For all of the stimuli, subjects were asked to answer a simple question presented at the beginning of the experiment. "Did a female perform the action in the sentence?', The sentences were digitized and presented by computer, and a voice reaction time device triggered by the subjects' response to the probe stopped the computer's clock. This sentence comprehension task was performed alone; during concurrent performance of a less demanding secondary task (finger tapping); and during concurrent performance of a more demanding secondary task, recognition span, that required subjects to match two consecutive presentations of digit sequences or spatially arrayed dots. We determined in advance the recognition span level of each patient so that we could equate task difficulty across individuals on the secondary task. Moreover, PD patients and control subjects were equally accurate and rapid at answering questions about simple sentences, thus equating for performance on the baseline sentence comprehension task. We report below our preliminary analyses of 17 very mild PD patients. 
To analyze our data, we set a threshold for a processing impairment at $20 \%$ slowing in response latency on an experimental condition compared to a control condition. During sentence comprehension without a secondary task, greater than a $20 \%$ slowing in response latency for object relative sentences compared to subject-relative center-embedded sentences was seen in $21 \%$ of PD patients. During their comprehension of simple sentences, $20 \%$ slowing for the recognition span condition compared to the finger tapping condition was seen in $21 \%$ of PD patients. However, $79 \%$ of PD patients showed greater than a $20 \%$ slowing in response latency as a function of the combined effects of the most demanding phrase structure and the most demanding secondary task. There was a corresponding increase in sentence comprehension errors (up to $15 \%$ more errors) even for these very mild PD patients.

These preliminary findings suggest that sentence processing interacts with resource demands. As cognitive resources became exhausted, PD patients appeared to encounter greater difficulty understanding sentences. Specifically, during the appreciation of simple sentences without concurrent secondary task performance, comprehension was essentially normal: resources remained available and responses to sentences remained rapid and accurate. With the increase of cognitive resource demands, performance was mildly degraded. However, performance deteriorated considerably under the condition where the center-embedded phrase of a sentence required an atypical syntactic-thematic mapping and a resource-demanding secondary task was performed concurrently.

\section{NEUROTRANSMITTER STUDIES OF SENTENCE PROCESSING IN PARKINSON'S DISEASE}

The etiology of Parkinson's disease has been elusive. Nevertheless, the final common pathway for the clinical disorder of PD is expressed largely through the degeneration of DA-containing neurons in the pars compacta of the substantia nigra. The clinical manifestations of PD thus can first be seen following the depletion of about $75 \%$ of DA neurons from this pigmented region in the rostral brainstem.

Several techniques have been used to examine the role of DA in cognitive functioning more closely. For example, investigators have attempted to correlate dopaminergic medication dosage with neuropsychological performance. The findings of these studies have been inconsistent (Loranger, Goodell, McDowell, Lee, \& Sweet, 1972; Lees \& Smith, 1983; Hietanen \& Teravainen, 1988; Cooper, Sagar, \& Sullivan, 1993; Brown, Marsden, Quinn, \& Wyke, 1984), although one recent study has found improvement in working memory performance following treatment of drug-naive PD patients with levodopa (Cooper et al., 1992). In our studies of sentence processing in Parkinson's disease, correlations of drug dosage with performance on neuropsychological tasks have been largely negative. 
Another strategy for establishing the role of DA in cognitive functioning has been to examine control subjects following a dosage of a dopaminergic agonist. Two studies have now shown that there may be significant improvement in working memory performance under conditions of DA agonist support (Luciana, Depue, Arbisi, \& Leon, 1992; Kimberg, D’Esposito, \& Farah, 1994). In the latter study, performance improved in subjects with baseline working memory that was less than the median, but worsened in subjects with baseline working memory greater than the median of our sample. This "inverted-U' response of cognitive functioning to dopaminergic supplementation in humans has been described as well in single-cell studies of DA metabolism in the dorsolateral frontal cortex of primates during working memory functioning (Williams \& Goldman-Rakic, 1995).

A final strategy has been to examine PD patients who are "on"' and "off', their DA supplementation. Specifically, augmentation of the DA neurotransmitter system in PD is most commonly accomplished with a medication that includes levodopa as the active ingredient. Levodopa is the precursor of dopamine. The bioavailability of levodopa is limited, typically less than $4 \mathrm{~h}$ (Wooten, 1987). As the CNS effect of the medication wears off, the features of PD patients' movement disorder become more apparent. We can take advantage of the predictably brief availability of levodopa to study cognitive functioning under conditions of relative DA depletion while we monitor the severity of the motor disorder that reflects CNS DA levels. For example, previous studies have demonstrated increased difficulty on measures of memory and attention when PD patients are relatively "off", their levodopa (Taylor, Saint-Cyr, \& Lang, 1987; Perry et al., 1985; Jahanshahi, Brown, \& Marsden, 1992; Mohr, Fabbrini, \& Williams, 1987), as indicated by the worsening of their motor disorder. However, such a decrement of cognitive functioning while "'off', levodopa has not been a universal finding (Pullman, Watts, Juncos, \& Sanes, 1990; Gotham, Brown, \& Marsden, 1988; Girotti et al., 1986).

In the following preliminary study, we have begun to assess sentence comprehension and working memory in 20 very mild (overwhelmingly Hoehn and Yahr Stage 1) PD patients fully supplemented by their dopamine medication, compared with their own performance on the identical tasks when they have been without DA supplementation for at least $12 \mathrm{~h}$. The patients were assessed under the two medication conditions on two separate occasions separated by about 4 weeks, and the order of assessment was randomized. PD patients performed a modified version of an oral sentence comprehension task (Grossman, Carvell, Stern, Gollomp, \& Hurtig, 1992). They also performed a sentence-picture matching task using orally presented sentences. Both measures used stimulus sentences containing right-branching subordinate and center-embedded subordinate phrases that were semantically unconstrained. To test for a nonspecific short-term memory effect, half of each type of sentence in both measures was lengthened with filler adjectives. Patients 
responded to the oral sentence comprehension task by selecting one of two choices; for the sentence-picture matching task, patients responded by selecting one of four picture choices that were made available. The PD patients also performed a dual task procedure where the primary task involved a reaction time measure for detecting the appearance of a target circle in a random position on a computer screen. The secondary tasks included counting from 1 to 10, a task of low resource demands, and performing a digit span task at the patient's own digit span ceiling, a task with greater resource demands.

An assessment of individual patient performance profiles revealed that 10 $(50 \%)$ of the PD patients demonstrate a center-embedded subordinate $<$ right-branching subordinate order of phrase structure difficulty under the "off" condition that was not evident under the "on" condition for the oral sentence comprehension task. We assessed the generalization of this finding to the sentence-picture matching task. We compared performance of these PD patients with the remaining 10 PD patients using a normalized difference score that was designed to reflect their performance for phrase structure (i.e., [(center-embedded - right-branching subordinate)/right-branching subordinate]), and for sentence length (i.e., [(adjective - no adjective)/no adjective]). A MANOVA with a group (grammatical impairment, no grammatical impairment) $\times$ sentence feature (phrase structure, sentence length) $\times$ drug status (on, off) $\times$ task (oral comprehension, sentence-picture match) design revealed a significant interaction effect for group $\times$ sentence feature $\times$ drug status $[F(1,18)=22.54 ; p<.0001]$. Subsequent comparisons, summarized in Fig. 4, revealed that PD patients with a sentence comprehension impairment are significantly impaired in their oral sentence comprehension performance for sentences with center-embedded subordinate phrases in comparison to the remaining PD patients when "off'" their medication $[t(18)=$ 3.38; $p<.003$ ]. However, these PD patient subgroups did not differ in their comprehension of center-embedded sentences when "on" their medication $[t(18)=0.39$; ns $]$, nor did they differ in their comprehension of rightbranching subordinate sentences when "off", their medication $[t(18)=1.42$; $\mathrm{ns}$ ]. In the sentence-picture matching task, the PD patients with a sentence comprehension impairment were significantly more compromised than the remaining PD patients when "off" their medication for sentences containing a center-embedded subordinate phrase $[t(18)=2.63 ; p<.01]$. However, these PD patient subgroups did not differ when "on" their medication for right-branching sentences $[t(18)=0.20 ; n s]$. Dopamine status had no effect on PD patients' comprehension of sentences that are different lengths.

Correlation analyses were used to relate sentence comprehension performance to working memory functioning in the "on"' and "off', state. These findings revealed a significant correlation between a normalized measure of span performance reflecting the difference between "on', and "off'" conditions (the difference between dual task performance with a demanding sec- 


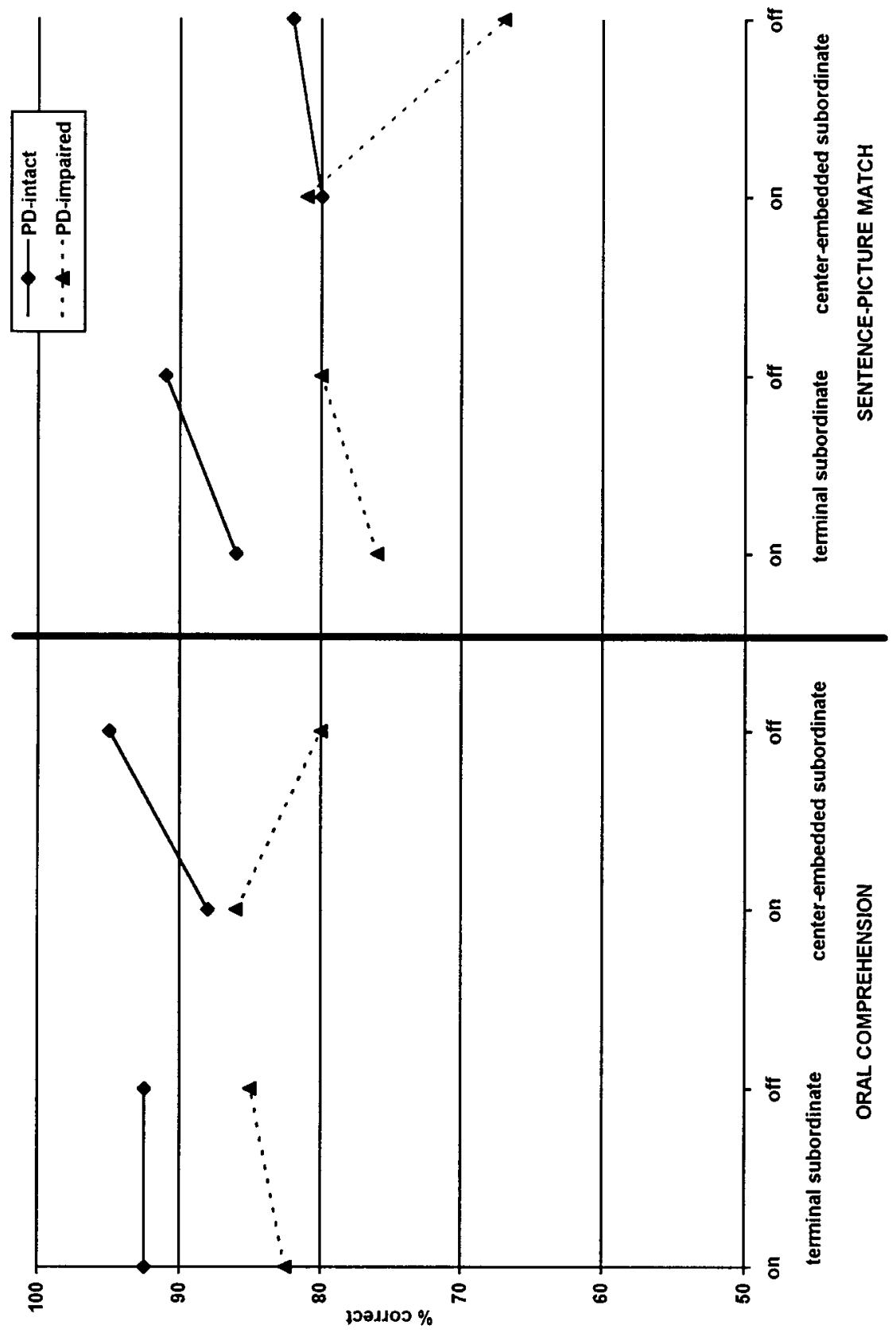


ondary task and performance of the baseline task alone, normalized to the baseline task) and a normalized measure of sentence comprehension reflecting the difference between "on" and "off" conditions (the difference between center-embedded subordinate sentences and right-branching subordinate sentences, normalized to right branching subordinate sentences) $[r(18)=0.437 ; p<.05]$. Greater difficulty with sentence comprehension during the "off" condition thus was associated with worse performance on a dual-task measure during the "off" condition.

These preliminary findings indicate that demanding aspects of sentence processing were selectively influenced by DA status in a subgroup of PD patients with impaired sentence comprehension. Moreover, there appeared to be a correlation between DA-dependent working memory performance and DA-dependent sentence comprehension in PD. The pattern of findings we observed is consistent with the hypothesis that reductions in brain DA levels limit the distribution of cognitive resources during sentence processing.

\section{CORTICAL AND SUBCORTICAL DYSFUNCTION IN THE SENTENCE PROCESSING IMPAIRMENTS OF PARKINSON'S DISEASE}

Dopaminergic neurons in the pars compacta of the substantia nigra are subdivided into smaller populations with relatively discrete projection zones. Lateral aspects of the substantia nigra project largely to the striatum, a collection of subcortical gray structures deep in the brain adjacent to the lateral ventricles. This nigro-striatal pathway is thought to play a crucial role in the movement disorder that figures so prominently in the clinical presentation of Parkinson's disease. A mesocortical projection system originates in the ventral tegmental area that is the most mesial aspect of the substantia nigra. This system projects predominantly to the mesial, dorsolateral, and orbital portions of the frontal lobe as well as to anterior temporal structures. These frontal regions form part of a complex, nonmotor anatomical loop that includes reciprocal projections with the striatum, as well as additional connections with the globus pallidus, the thalamus, and eventually back to frontal cortices (Alexander \& Crutcher, 1990). The dissociable nature of these motor and nonmotor DA projection systems is demonstrated by the finding that we have not observed significant correlations between sentence interpretation accuracy and the severity or laterality of motor performance. We have hypothesized that interruption of the fronto-striatal system plays a crucial role in the sentence processing deficits of PD patients.

One strategy to test hypotheses about the anatomical basis of the cognitive

FIG. 4. Oral sentence comprehension and sentence-picture matching for sentences containing terminal subordinate phrases and center-embedded phrases in Parkinson's disease patients "on" and "off"' their dopaminergic medication. 
deficits in PD would be to correlate volumetric measures of brain structures with cognitive difficulty. We have not been able to identify specific anatomical regions that have atrophied with sufficient reliability in nondemented PD patients to be detected by MRI imaging techniques. Nor have we been able to detect reliable modifications in the substantia nigra pigmenatation patern of PD patients with MRI (Stern, Braffman, Skolnick, Hurtig, \& Grossman, 1989).

An alternative approach has been to use functional imaging to relate measures of regional brain activity to the cognitive deficits of PD patients. Early studies were able to identify areas of altered brain activity in PD at rest with positron emission tomography (PET), including the striatum and frontal cortex (Wolfson, Leenders, Brown, \& Jones, 1985; Perlmutter \& Raichle, 1985). However, early attempts to relate changes in functional brain activity such as these to cognitive deficits were generally negative, due in large part to our poor understanding of the role played by these brain regions in cognitive functioning (Kuhl, Metter, \& Riege, 1984; Globus, Mildworf, \& Melamed, 1985). For example, PD patients studied with single photon emission computed tomography (SPECT) were asked to perform a battery of neuropsychological tasks from which a laterality index was derived. Little relationship was found between the laterality of cognitive functioning and asymmetries on the SPECT studies (Agniel et al., 1991). More recently, correlative studies motivated by the cognitive neuroscience literature have identified important relationships between defects in regional brain activity and specific cognitive difficulties. Jagust, Reed, Martin, Eberling, \& Nelson-Abbott (1992) thus have found significant correlations between PD patients' performance of executive measures and dorsolateral frontal cortex activity as measured by SPECT.

We specifically sought to determine whether the sentence comprehension impairment in PD is due to the interruption of a fronto-striatal loop. To accomplish this, we related defects in cerebral glucose metabolism obtained at rest with PET to the results of sentence comprehension studies performed $1 \mathrm{~h}$ prior to the PET scan (Grossman et al., 1993). We observed a significant correlation between bilateral mesial frontal cortex and comprehension of sentences with center-embedded subordinate phrases, but no correlation with the comprehension of simple sentences. We also found significant correlations between glucose metabolism in left mesial frontal cortex and performance on a sentence judgment task where an obligatory grammatical morpheme was omitted or its phonological shape was changed. Linear regression analyses related overall sentence comprehension to the left mesial frontal region and the left caudate; the comprehension of sentences with center-embedded phrases was related to the left mesial frontal region and the left dorsolateral frontal region; the detection of an omitted grammatical morpheme was related to the left mesial frontal region and the left dorsolateral frontal region. 
These findings are consistent with the claim that the interruption of a frontostriatal network interferes with sentence processing in Parkinson's disease.

A recent advance has been the ability to assess PD patients' regional brain activity during a cognitive challenge. This is important because reductions in brain activity at rest are difficult to interpret: It may be that a resting brain defect is due to diaschisis, and that a defective brain region at rest can be recruited normally when challenged. A PET activation study thus has defined recruitment of the motor system in controls during a simple motor task, and found that dorsolateral frontal cortex also was recruited when subjects were asked to perform an internally generated random motor sequence (Playford, Jenkins, \& Passingham, 1992). In this context, the investigators found reduced recruitment of dorsolateral frontal cortex in PD during the performance of the internally generated random motor sequence.

We have performed activation studies of neurologically intact control subjects while processing sentences, comparing their pattern of regional cerebral recruitment with that seen in PD patients (Grossman et al., 1992). Sentences containing simple transitive action verbs flanked by noun phrases containing a male name and a female name were presented. Half of the sentences contained an adjective and half had an atypical syntactic-thematic mapping (e.g., passive voice, object-relative center-embedded sentence). In one challenge, subjects were asked to detect whether a sentence contained an adjective, an assessment of grammatical form class; in a second challenge, subjects were asked to detect whether a female performed the action described in the sentence, an assessment of thematic role. The baseline condition probed subjects' ability to detect the presence of the letter " $\mathrm{K}$ ', in the sentence. We used identical stimulus sentences for these three challenges, manipulating only the specific nature of the probe.

Control subjects demonstrated significant recruitment of left dorsolateral and inferior frontal cortex, the anterior cingulate bilaterally, left caudate, and left thalamus during the detection of an adjective. Increased activity was also seen in left posterior superior temporal regions during the detection of an agent. The recruitment of mesial frontal regions has been associated with selective attention in several PET activation studies (Pardo, Pardo, Janer, \& Raichle, 1990; Corbetta, Miezin, Dobmeyer, Shulman, \& Petersen, 1991), and this has been confirmed by the results of lesion studies (Rubens, 1975; Bogousslavsky \& Regli, 1990; Alexander \& Schmitt, 1980). Control subjects also activated left inferior frontal cortex that many lesion studies have related to grammatical processing (Novoa \& Ardila, 1987; Nadeau, 1988; Mohr, 1976; Alexander, Naeser, \& Palumbo, 1990; Alexander, Benson, \& Stuss, 1989). Caplan and co-workers have demonstrated a similar distribution of regional frontal activity with a PET activation study of grammatical processing (Stromswold, Caplan, Alpert, \& Rauch, 1996; Caplan, 1994). Dorsolateral frontal cortex recruitment has been associated with working memory 
(D'Esposito et al., 1995). PD patients were unable to recruit these frontal and caudate areas during similar grammatical challenges, although there was some increased posterior superior temporal activity during the thematic role challenge. These findings suggest that PD patients have difficulty recruiting a network of specific brain regions associated with working memory during a sentence comprehension challenge.

\section{GENERAL DISCUSSION}

Patients with Parkinson's disease have significant impairments in their sentence comprehension performance. We believe that these deficits are multifactorial in nature. Factors related to processing the syntactic structure of a sentence contribute importantly to sentence interpretation, and the processing of fundamental grammatical components for sentence interpretation such as these appears to be compromised in some PD patients (Lieberman et al., 1992; Grossman et al., 1994). However, the implementation of these processes apparently does not come for free, but occurs at a computational cost. Simpler grammatical forms - often the default options of a sentence comprehension system - are processed in a virtually effortless and transparent fashion. More demanding grammatical forms are processed at a greater price. The associated computational cost can be reduced by making available explicit cues to the grammatical attributes of a sentence, such as surface markers for the passive voice. Other complex sentences featuring implicit structural information require greater cognitive resources to support processing. Most PD patients appear to be impaired at implementing the resources necessary to support the processing of more complex sentences. Future research will need to address more precisely the nature of these processing resources and how they are compromised in PD. Nevertheless, these findings in PD support the claim that working memory resources contribute importantly to sentence processing such as the mental manipulation of information required to interpret syntactic-thematic relations.

The physiologic basis for this sentence processing component can be established in part from these studies as well. Our preliminary findings assessing the role of DA in sentence interpretation thus have begun to show that PD patients depleted of their dopamine medication are selectively impaired in their sentence processing. Our preliminary findings appear to relate DA to the processing resources needed to compute demanding aspects of sentences, although CNS levels of DA appear to have virtually no impact on a shortterm memory slave buffer. Finally, PET studies of regional cerebral activity in PD have shown that left dorsolateral and mesial frontal cortical and striatal activity correlates with sentence processing, and that PD patients are limited in their ability to recruit these brain regions during a sentence comprehension challenge.

To be sure, there are many shortcomings associated with these studies. 
We have recruited PD patients from a university clinic specializing in the diagnosis and treatment of movement disorders, and this may have inadvertently biased our sampling of patients. The vast majority of PD patients we have studied were mildly impaired and not demented. This has limited our ability to generalize our findings to patients who are more severely impaired and leaves open the possibility that other cognitive deficits may limit language function in more impaired patients. We have performed within-subject analyses to demonstrate selective deficits in PD patients, but direct comparisons with control subjects have been limited at times by a ceiling effect. We have examined a variety of sentence comprehension factors such as phrase structure and grammatical voice, but there are clearly additional aspects of sentence processing that remain to be assessed in order to establish more precisely the scope of the sentence processing deficit in PD. The number of PD patients studied with PET activation has been limited, and we have administered only one sentence processing procedure to these patients, so our results remain to be confirmed by additional studies. Similarly, our preliminary assessment of the relationship between DA metabolism and sentence processing has been quite limited. With these caveats in mind, we conclude that PD patients have a sentence processing impairment that is multifactorial in nature, involving at least the distribution of cognitive resources as well as the appreciation of grammatical attributes, and we attribute these impairments to the interruption of a DA-mediated fronto-striatal network that contributes to sentence processing.

\section{REFERENCES}

Agniel, A., Celsis, P., Viallard, G., Montastrue, J. L., Rascol, O., Demonet, J. F., MarcVergnes, J. P., \& Rascol, A. 1991. Cognition and cerebral blood flow in lateralised Parkinsonism: Lack of functional lateral asymmetries. Journal of Neurology, Neurosurgery, and Psychiatry, 54, 783-786.

Alexander, G. E., \& Crutcher, M. D. 1990. Functional architecture of basal ganglia circuits: Neural substrates of parallel processing. Trends in Neuroscience, 13, 266-271.

Alexander, M. P., Benson, D. F., \& Stuss, D. T. 1989. Frontal lobes and language. Brain and Language, 37, 656-691.

Alexander, M. P., Naeser, M. A., \& Palumbo, C. 1990. Broca's area aphasia: Aphasia after lesions including the frontal operculum. Neurology, 40, 353-362.

Alexander, M. P., \& Schmitt, M. A. 1980. The aphasia syndrome that follows stroke in the left anterior cerebral artery territory. Archives of Neurology, 37, 97-100.

Auriacombe, S., Grossman, M., Carvell, S., Gollomp, S., Stern, M. B., \& Hurtig, H. I. 1993. Verbal fluency deficits in Parkinson's disease. Neuropsychology, 7, 182-192.

Baddeley, A., Logie, R., Bressi, S., Della Sala, S., \& Spinnler, H. 1986. Dementia and working memory. The Quarterly Journal of Experimental Psychology A, 38, 603-618.

Bogousslavsky, J., \& Regli, F. 1990. Anterior cerebral artery territory infarction in the Lausanne registry. Archives of Neurology, 47, 144-150.

Brown, R. G., \& Marsden, C. D. 1984. How common is dementia in Parkinson's disease? Lancet, 2, 1262-1265. 
Brown, R. G., \& Marsden, C. D. 1988a. An investigation of the phenomenon of "set" in Parkinson's disease. Movement Disorders, 3, 152-161.

Brown, R. G., \& Marsden, C. D. 1988b. Internal versus external cues and the control of attention in Parkinson's disease. Brain, 111, 325-345.

Brown, R. G., \& Marsden, C. D. 1988c. Neuropsychology and cognitive function in Parkinson's disease: An overview. In C. D. Marsden \& S. Fahn (Eds.), Movement disorders 2 (pp. 99-123). London: Butterworths.

Brown, R. G., Marsden, C. D., Quinn, N., \& Wyke, M. A. 1984. Alterations in cognitive performance and affect arousal state during fluctuations in motor function in Parkinson's disease. Journal of Neurology, Neurosurgery, and Psychiatry, 47, 454-465.

Caplan, D. 1994. The cognitive neuroscience of syntactic processing. In M. S. Gazzaniga (Ed.), The cognitive neurosciences (pp. 871-879). Cambridge: MIT Press.

Caplan, D., \& Waters, G. S. 1990. Short-term memory and language comprehension: A critical review of the neuropsychological literature. In G. Vallar \& T. Shallice (Eds.), Neuropsychological impairments of short-term memory (pp. 337-389). Cambridge: Cambridge Univ. Press.

Caramazza, A., Basili, A. G., Koller, J. J., \& Berndt, R. S. 1981. An investigation of repetition and language processing in a case of conduction aphasia. Brain and Language, 14, 235271.

Cooper, J. A., Sagar, H. J., Doherty, S. M., Jordan, N., Tidswell, P., \& Sullivan, E. V. 1992. Different effects of dopaminergic and anticholinergic therapies on cognitive and motor function in Parkinson's disease. Brain, 115, 1701-1725.

Cooper, J. A., Sagar, H. J., Jordan, N., Harvey, N. S., \& Sullivan, E. V. 1991. Cognitive impairment in early, untreated Parkinson's disease and its relationship to motor disability. Brain, 114, 2095-2122.

Cooper, J. A., Sagar, H. J., \& Sullivan, E. V. 1993. Short-term memory and temporal ordering in early Parkinson's disease: Effects of disease chronicity and medication. Neuropsychologia, 31, 933-949.

Corbetta, M., Miezin, F. M., Dobmeyer, S., Shulman, G. L., \& Petersen, S. E. 1991. Selected and divided attention during visual discriminations of shape, color, and speed. Journal of Neuroscience, 11, 2383-2402.

Cummings, J. L., Darkins, A., Mendez, M., Hill, M. A., \& Benson, D. F. 1988. Alzheimer's disease and Parkinson's disease: comparison of speech and language alterations. Neurology, 38, 680-684.

D’Esposito, M., Detre, J. A., Alsop, D. C., Shin, R. K., Atlas, S. W., \& Grossman, M. 1995. The neural basis of the central executive system of working memory. Nature, 378, 279281.

Darkins, A. W., Fromkin, V. A., \& Benson, D. F. 1988. A characterization of the prosodic loss in Parkinson's disease. Brain and Language, 34, 315-327.

Ebmeier, K. P., Calder, S. A., Crawford, J. R., Stewart, L., Cochrane, R. H. B., \& Besson, J. A. O. 1991. Dementia in idiopathic Parkinson's disease: prevalence and relationship with symptoms and signs of Parkinsonism. Psychological Medicine, 21, 69-76.

Geyer, H., \& Grossman, M. 1994. Investigating the basis for the sentence comprehension deficit in Parkinson's disease. Journal of Neurolinguistics, 8, 191-205.

Girotti, F., Carella, F., Grassi, M. P., Soliveri, P., Marano, R., \& Caraceni, T. 1986. Motor and cognitive performances of Parkinsonian patients in the on and off phases of the disease. Journal of Neurology, Neurosurgery, and Psychiatry, 49, 657-660.

Globus, M., Mildworf, B., \& Melamed, E. 1985. Cerebral blood flow and cognitive impairment in Parkinson's disease. Neurology, 35, 1135-1139. 
Gotham, A. M., Brown, R. G., \& Marsden, C. D. 1988. 'Frontal' cognitive function in patients with Parkinson's disease 'on' and 'off' levodopa. Brain, 111, 299-321.

Grossman, M. 1993. Short-term memory in aphasia. In G. Blanken, J. Dittmann, H. Grimm, J. C. Marshall, \& C.-W. Wallesch (Eds.), Linguistic disorders and pathologies: An international handbook (pp. 332-351). Berlin: de Gruyter.

Grossman, M., Carvell, S., Gollomp, S., Stern, M. B., Reivich, M., Morrison, D., Alavi, A., \& Hurtig, H. I. 1993. Cognitive and physiological substrates of impaired sentence processing in Parkinson's disease. Journal of Cognitive Neuroscience, 5, 480-498.

Grossman, M., Carvell, S., Gollomp, S., Stern, M. B., Vernon, G., \& Hurtig, H. I. 1991. Sentence comprehension and praxis deficits in Parkinson's disease. Neurology, 41, 16201626.

Grossman, M., Carvell, S., \& Peltzer, L. 1994. The sum and substance of it: The appreciation of mass and count quantifiers in Parkinson's disease. Brain and Language, 44, 351-384.

Grossman, M., Carvell, S., Stern, M. B., Gollomp, S., \& Hurtig, H. I. 1992. Sentence comprehension in Parkinson's disease: The role of attention and memory. Brain and Language, 42, 347-384.

Grossman, M., Crino, P., Stern, M. B., Reivich, M., \& Hurtig, H. I. 1992. Attention and sentence processing deficits in Parkinson's disease: The role of anterior cingulate. Cerebral Cortex, 2, 513-525.

Grossman, M., Stern, M. B., Gollomp, S., Vernon, G., \& Hurtig, H. I. 1994. Verb learning in Parkinson's disease. Neuropsychology, 8, 413-423.

Hietanen, M., \& Teravainen, H. 1988. Dementia and treatment with I-dopa in Parkinson's disease. Movement Disorders, 3, 263-270.

Illes, J. 1990. Neurolinguistic features of spontaneous language production dissociate three forms of neurodegenerative disease: Alzheimer's, Huntington's, and Parkinson's. Brain and Language, 37, 628-642.

Illes, J., Metter, E. J., Hanson, W. R., \& Iritani, S. 1988. Language production in Parkinson's disease: Acoustic and linguistic considerations. Brain and Language, 33, 146-160.

Jagust, W. J., Reed, B. R., Martin, E. M., Eberling, J. L., \& Nelson-Abbott, R. A. 1992. Cognitive function and regional cerebral blood flow in Parkinson's disease. Brain, 115, 521-537.

Jahanshahi, M., Brown, R. G., \& Marsden, C. D. 1992. The effect of withdrawal of dopaminergic medication on simple and choice reaction time and the use of advance information in Parkinson's disease. Journal of Neurology, Neurosurgery, and Psychiatry, 58, 11681176.

Jordan, N., Sagar, H. J., \& Cooper, J. A. 1992. Cognitive components of reaction time in Parkinson's disease. Journal of Neurology, Neurosurgery, and Psychiatry, 55, 658-664.

Just, M. A., \& Carpenter, P. A. 1992. A capacity theory of comprehension: Individual differences in working memory. Psychological Review, 99, 122-149.

Kimberg, D. Y., D’Esposito, M., \& Farah, M. J. 1994. The effects of bromocriptine, a D2 dopamine receptor agonist, on the cognitive abilities of human subjects with different working memory capacities. Society for Neuroscience Abstracts, 20, 1271.

Kuhl, D. E., Metter, E. J., \& Riege, W. H. 1984. Patterns of local cerebral glucose utilization determined in Parkinson's disease by the $\left[{ }^{18} \mathrm{~F}\right]$ fluorodexyglucose method. Annals of Neurology, 15, 419-424.

Lees, A. J., \& Smith, E. 1983. Cognitive deficits in the early stages of Parkinson's disease. Brain, 106, 257-270.

Levin, B. 1993. English verb classes and alternations. Chicago: Univ. of Chicago Press. 
Lieberman, P., Friedman, J., \& Feldman, L. S. 1990. Syntax comprehension in Parkinson's disease. Journal of Nervous and Mental Disease, 178, 360-366.

Lieberman, P., Kako, E., Friedman, J., Tajchman, G., Feldman, L. S., \& Jiminez, E. B. 1992. Speech production, syntax comprehension, and cognitive deficits in Parkinson's disease. Brain and Language, 43, 169-189.

Loranger, A. W., Goodell, H., McDowell, F. H., Lee, J. E., \& Sweet, R. D. 1972. Intellectual impairment in Parkinson's syndrome. Brain, 95, 405-412.

Luciana, M., Depue, R. A., Arbisi, P., \& Leon, A. 1992. Facilitation of working memory in humans by a D2 dopamine receptor agonist. Journal of Cognitive Neuroscience, 4, 5868.

Luria, A. R., Sokolov, E. N., \& Klimkowski, M. 1967. Toward a neurodynamic analysis of memory disturbances with lesions of the left temporal lobe. Neuropsychologia, 5, 1-11.

Matison, R., Mayeux, R., Rosen, J., \& Fahn, S. 1982. "Tip-of-the-tongue”, phenomenon in Parkinson's disease. Neurology, 32, 567-570.

Mayeux, R., Denaro, J., Hemenegildo, N., Marder, K., Tang, M.-X., Cote, L. J., \& Stern, Y. 1992. A population-based investigation of Parkinson's disease with and without dementia. Archives of Neurology, 49, 492-497.

Mayeux, R., Stern, Y., Rosenstein, R., Marder, K., Hauser, A., Cote, L., \& Fahn, S. 1988. An estimate of the prevalence of dementia in idiopathic Parkinson's disease. Archives of Neurology, 45, 260-262.

McCarthy, R. A., \& Warrington, E. K. 1987a. The double dissociation of short-term memory for lists and sentences: Evidence from aphasia. Brain, 110, 1545-1563.

McCarthy, R. A., \& Warrington, E. K. 1987b. Understanding: A function of short-term memory? Brain, 110, 1565-1587.

Mohr, E., Fabbrini, G., \& Williams, J. 1987. Cognitive concomitants of dopamine system stimulation in parkinsonian patients. Journal of Neurology, Neurosurgery, and Psychiatry, 50, 1192-1196.

Mohr, J. P. 1976. Broca's area and Broca's aphasia. In H. Whitaker (Ed.), Studies in neurolinguistics (Vol. 1). New York: Academic Press.

Nadeau, S. 1988. Impaired grammar with normal fluency and phonology. Brain, 111, 11111137.

Natsopoulos, D., Katsarou, Z., Bostantzopoulos, S., Grouios, G., Mentenopoulos, G., \& Logothetis, J. 1991. Strategies in comprehension of relative clauses in Parkinsonian patients. Cortex, 27, 255-268.

Novoa, O. P., \& Ardila, A. 1987. Linguistic abilities in patients with prefrontal damage. Brain and Language, 30, 206-225.

Orsini, A., Fragassi, N. A., Chiacchio, L., \& Falanga, A. M. 1987. Verbal and spatial memory span in patients with extrapyramidal diseases. Perceptual and Motor Skills, 65, 555-558.

Owen, A. M., James, M., Leigh, P. N., Summers, B. A., Marsden, C. D., Quinn, N. P., Lange, K. W., \& Robbins, T. W. 1992. Fronto-striatal cognitive deficits at different stages of Parkinson's disease. Brain, 115, 1727-1751.

Pardo, J. V., Pardo, P. J., Janer, K. W., \& Raichle, M. E. 1990. The anterior cingulate cortex mediates processing selection in the Stroop attentional conflict paradigm. Proceedings of the National Academy of Sciences U.S.A., 87, 256-259.

Parkinson, J. 1817. An essay on the shaking palsy. London: Sherwood, Neely, and Jones.

Perlmutter, J. S., \& Raichle, M. E. 1985. Regional blood flow in hemiparkinsonism. Neurology, 35, 1127-1134.

Perry, E. K., Curtis, M., Dick, D. J., Candy, J. M., Atack, J. R., Bloxham, C. A., Blessed, G., 
Fairbairn, A., Tomlinson, B. E., \& Perry, R. H. 1985. Cholinergic correlates of cognitive impairment in Parkinson's disease: Comparisons with Alzheimer's disease. Journal of Neurology, Neurosurgery, and Psychiatry, 48, 413-421.

Pillon, B., Deweer, B., Agid, Y., \& Dubois, B. 1993. Explicit memory in Alzheimer's, Huntington's, and Parkinson's diseases. Archives of Neurology, 50, 374-379.

Playford, E. D., Jenkins, I. H., \& Passingham, R. E. 1992. Impaired mesial frontal and putamen activation in Parkinson's disease: A positron emission tomography study. Annals of Neurology, 32, 151-161.

Pullman, S. L., Watts, R. L., Juncos, J. L., \& Sanes, J. N. 1990. Movement amplitude choice reaction time performance in Parkinson's disease may be independent of dopaminergic status. Journal of Neurology, Neurosurgery, and Psychiatry, 53, 279-283.

Rubens, A. B. 1975. Aphasia with infarction in the territory of the anterior cerebral artery. Cortex, 11, 239-250.

Saffran, E. M., \& Martin, N. 1990. Short-term memory and sentence processing: A case study. In G. Vallar \& A. Baddeley (Eds.), Cognitive neuropsychology of short-term memory (pp. 189-221). Cambridge: Cambridge Univ. Press.

Stern, M. B., Braffman, B. H., Skolnick, B. E., Hurtig, H. I., \& Grossman, R. I. 1989. Magnetic resonance imaging in Parkinson's disease and parkinsonian syndromes. Neurology, 39, $1524-1526$.

Stromswold, K., Caplan, D., Alpert, N., \& Rauch, S. 1996. Localization of syntactic comprehension by positron emission tomography. Brain and Language, 52, 452-473. (abstract)

Taylor, A. E., Saint-Cyr, J. A., \& Lang, A. E. 1987. Parkinson's disease: Cognitive changes in relation to treatment response. Brain, 110, 35-51.

Taylor, A. E., Saint-Cyr, J. A., \& Lang, A. E. 1990. Memory and learning in early Parkinson's disease. Brain and Cognition, 2, 211-232.

Warrington, E. K., \& Shallice, T. 1969. The selective impairment of auditory verbal shortterm memory. Brain, 92, 885-896.

Williams, G. V., \& Goldman-Rakic, P. S. 1995. Modulation of memory fields by dopamine D1 receptors in prefrontal cortex. Nature, 376, 572-575.

Wolfson, L. I., Leenders, K. L., Brown, L. L., \& Jones, T. 1985. Alterations of regional cerebral blood flow and oxygen metabolism in Parkinson's disease. Neurology, 35, 1399-1405.

Wooten, G. F. 1987. Pharmacokinetics of levodopa. In C. D. Marsden \& S. Fahn (Eds.), Movement disorders 2 (pp. 231-248). London: Butterworths. 